\title{
Procurement and Ex-Situ Perfusion of Isolated Slaughterhouse-Derived Livers as a Model of Donors after Circulatory Death
}

\author{
Daniele Dondossola1,2, Stefano De Falco ${ }^{3}$, Alessia Kersik1, Marco Maggioni4, Luca Di Girolamo3, \\ Osvaldo Biancolilli ${ }^{3}$, Mattia Busana ${ }^{3}$, Caterina Lonati ${ }^{3,5}$, Francesco Carù ${ }^{5}$, Alberto Zanella ${ }^{2,3}$ and \\ Stefano Gatti ${ }^{5}$ \\ ${ }^{1}$ General and Liver Transplant Surgery Unit, Fondazione IRCCS Ca' Granda, Ospedale Maggiore Policlinico, Milan, Italy; ${ }^{2}$ Department of \\ Pathophysiology and Transplantation, Università degli Studi di Milano, Milan, Italy; ${ }^{3}$ Department of Anesthesia and Critical Care, Fondazione IRCCS \\ Ca' Granda, Ospedale Maggiore Policlinico, Milan, Italy; ${ }^{4}$ Pathology Department, Fondazione IRCCS Ca' Granda, Ospedale Maggiore Policlinico, \\ Milan, Italy; ${ }^{5}$ Center for Preclinical Research, Fondazione IRCCS Ca' Granda, Ospedale Maggiore Policlinico, Milan, Italy
}

\begin{abstract}
Ex-situ machine perfusion (MP) techniques are increasingly used in clinical settings, especially on grafts from donors after cardiac death (DCD). However, the biological effects elicited by machine perfusion are largely unknown, and a substantial number of animal studies are presently focused on this topic. The aim of the present study was to describe a model of DCD based on ex-situ perfusion of liver grafts derived from animals used for food production.

Procurement took place within a slaughterhouse facility. After cold storage, the liver grafts were perfused with autologous blood-enriched perfusion fluid in a clinically fashioned closed circuit normothermic MP (NMP). During the rewarming phase, temperature and flows were progressively increased to reach target values. Perfusate and tissue samples were collected to assess NMP functionality. Grafts were classified as transplantable (LT-G) or not (nLT-G) according to clinical criteria, and viability was confirmed by histopathological analysis.

Four grafts were classified as LT-G and three as nLT-G at the end of NMP. Histology confirmed the absence of major damage in LT-G, while nLT-G presented diffuse necrosis. Interestingly, an early impairment of the hepatocyte respiratory chain, leading to cell necrosis and graft non-viability, was documented in nLT-G for the first time. These parameters, together with indocyanine-green dye and citrate clearance could contribute to graft evaluation in clinical settings. The model provides a promising and reproducible method to replace dedicated experimental animals in research on machine perfusion of DCD grafts in line with the 3 Rs principles.
\end{abstract}

\section{Introduction}

Over the last few years, the increased number of patients requiring organ transplantation has caused a challenging organ shortage as the number of suitable donors has remained relatively stable. In 2017, according to the annual report of the Scientific Registry of Transplant Recipients (SRTR), 1334 patients died on the waiting list for a liver in the USA, while 703 liver grafts were not transplanted (Kim et al., 2019).

To overcome this shortage, liver grafts from high risk donors (also called extended criteria donors, ECD) are increasingly used although such grafts generally show a poorer outcome after liver transplantation (LT) (Vodkin and Kuo, 2017). Indeed, an in- creased risk of primary non-function (PNF), initial poor function (IPF), and long-term worse outcome are associated with ECD liver grafts (López-Navidad and Caballero, 2003; Feng et al., 2006; Dondossola et al., 2017a). Among ECDs, donors after cardiocirculatory death (DCD), in particular type II DCD - unexpected cardiac arrest with unsuccessful resuscitation (Thuong et al., 2016) - represent a major organ source with a significant potential to increase the number of donations (Blackstock and Ray, 2014; Manyalich et al., 2018). However, DCD can have detrimental effects on post-transplant survival and quality of life. For this reason, there is a high discard rate of these grafts. The DCD-related problems mainly stem from prolonged warm ischemia time (WIT), which reduces the tissue energy pool leading to cell death (Merlen et al.,
Received September 13, 2019; Accepted December 9, 2019;

Epub December 12, 2019; ( The Authors, 2019.

ALTEX 37(2), 243-254. doi:10.14573/altex.1909131

Correspondence: Dondossola Daniele, MD

Fondazione IRCCS Ca' Granda Ospedale Maggiore Policlinico

Via Franscesco Sforza, 35 - 20122 Milan, Italy

(daniele.dondossola@policlinico.mi.it)
This is an Open Access article distributed under the terms of the Creative Commons Attribution 4.0 International license (http://creativecommons.org/licenses/by/4.0/) which permits unrestricted use, distribution and reproduction in any medium, provided the original work is appropriately cited. 
2019). However, although the clinical impact of WIT is generally accepted, only little data on the subcellular mechanisms underlying its consequences is available, and potential therapeutic targets are still unclear.

In an attempt to mitigate the detrimental impact of ECD and to reduce the discard rate of DCD, ex-situ perfusion systems have been re-introduced in clinical settings (Guarrera et al., 2010). These procedures consist of extracorporeal perfusion devices that allow continuous and dynamic perfusion of organs at a range of different set temperatures using different perfusion fluids. The aims of ex-situ perfusion studies have been to improve graft preservation, reduce ischemia-reperfusion injury, and evaluate viability of marginal grafts (Hessheimer and Fondevila, 2017; Selten et al., 2017). Following the demonstration of a clinical advantage of normothermic ex-situ perfusion (NMP) of selected grafts (Nasralla et al., 2018), concerns were raised on the potential metabolic and biomolecular processes elicited by these dynamic perfusions. Indeed, although NMP allows graft evaluation before transplantation, increasing safety and the acceptance rate, its full potential is presently underestimated due to uncertainty regarding its effects on the viability criteria.

Machine perfusion and DCD represent a significant emerging field of experimental and clinical research as demonstrated by approximately 500 publications over the last 10 years. Several groups have explored this subject using different methods and techniques. However, the lack of standardized models and the great differences in procurement procedures and perfusion variables reported negatively affect the translational impact of the results.

As in vitro studies cannot be used in this setting due to the absence of physiological cell-to-cell contact and environment (Daniel et al., 2018), in vivo models with dedicated experimental animals are commonly used to study NMP and DCDs. Only few investigations have tried to use commercial animals as a source of experimental liver grafts (Grosse-Siestrup et al., 2001, 2002b). Further, in these studies, the description of procurement procedures was not provided in detail, and technique reproducibility had some bias.

As further development of preclinical studies on machine perfusion and type II DCD is needed, standardized, easily accessible, inexpensive (in terms of life and economical resources) translational models should be developed. Large animal organs are preferred for the study of organ preservation, physiology and pharmacology. Indeed, a standardized model of NMP could be an effective bridge between in vitro and in vivo experiments. On the other hand, use of dedicated experimental animals to optimize the perfusion protocols does not appear to be justified according to the $3 \mathrm{R}$ principles.

Based on our experience in clinical and preclinical ex-situ perfusions (Bassani et al., 2016; Lonati et al., 2018; Roffia et al., 2018; Dondossola et al., 2018), we designed a study aiming to improve and standardize an ethical and cost-effective procurement/perfusion model using slaughterhouse-derived liver grafts. This model could help characterization of liver metabolism and viability during ex-situ perfusion and provide data that could be translated effectively to clinical settings.

\section{Animals, material and methods}

\section{Animals}

Liver grafts were obtained from Landrace pigs weighing 40-60 kg $(\mathrm{n}=8)$. Animals received adequate husbandry. The animals were slaughtered at an abattoir $30 \mathrm{~km}$ away from our preclinical facility for commercial purposes. Necessary measures were taken to minimize pain, distress and suffering during the slaughtering process in accordance with the Italian and EU laws (EC, 1099/2009). Pigs were killed by sudden induction of brain hemorrhage followed by terminal exsanguination according to Veterinary and Food Security Department regulations. After exsanguination, the pigs were immersed in hot water $\left(70^{\circ} \mathrm{C}\right)$ to remove hair and to clean the surface.

\section{Materials and instruments}

Surgical instruments, perfusion sets and fluids, ice, and organ boxes were transported to the slaughterhouse and kept sterile until procurement (Tab. S1, S2 ${ }^{1}$ ). Dialysis and perfusion fluids were stored at $4^{\circ} \mathrm{C}$. Personal protection devices consisted of disposable coveralls, surgical gloves, and protective glasses.

\section{Isolated organ perfusion systems}

The liver perfusion circuit consisted of an organ chamber, two roller pumps, a centrifugal pump, a reservoir, a membrane lung, and silicon tubing. The closed circuit with cannulated vena cava had the portal (PVP), arterial (HAP) and cava (CVP) pressure set at 8 $\mathrm{mmHg}, 75 \mathrm{mmHg}$ and $1 \mathrm{mmHg}$, respectively. The membrane lung and reservoir were equipped with a heat exchanger to control the perfusate temperature. Graft temperature during perfusion was set to $38^{\circ} \mathrm{C}$. Three syringe pumps were connected to enable infusion of drugs or fluids (Fig. 1).

\section{Perfusion fluid analysis}

Inflow and outflow perfusate composition was monitored every $15 \mathrm{~min}$ over the first hour and every $30 \mathrm{~min}$ thereafter (total perfusion time $240 \mathrm{~min}$ ). Perfusion fluid gas analyses and blood tests were performed.

\section{Mean perfusion fluid hemodynamic and metabolic measurements}

During the whole experiment, resistance in the venous system was calculated as mean PVP minus CVP ( $\mathrm{mmHg}$ ) divided by blood flow in the portal vein $(\mathrm{L} / \mathrm{min})$, while resistance in the hepatic artery was calculated as mean HAP minus CVP divided by blood flow in the hepatic artery. Oxygen consumption $\left(\dot{V O}_{2}\right)$ was measured using the modified Fick equation. Pre-liver perfusate samples, collected after the membrane lung, were designated $\mathrm{O}_{2}$ enriched perfusate (arterial blood of the Fick equation), whereas post-liver perfusate samples, collected directly from the IVC, were used for calculation of venous oxygen content in the Fick equation. Oxygen delivery $\left(\mathrm{DO}_{2}\right)$ was measured likewise (Banan et al., 2016).

$\mathrm{O}_{2}$ content of pre-liver perfusate $\left(\mathrm{C}_{P R E} \mathrm{O}_{2}\right)=[1.34 \times \mathrm{Hb}(\mathrm{g} / \mathrm{dL})$ PRE-LIVER $\left.\times \mathrm{HbO}_{2}(\%)\right]+0.003 \times \mathrm{P}_{\text {PRE-LIVER }} \mathrm{O}_{2}(\mathrm{mmHg})$

\footnotetext{
1 doi:10.14573/altex.1909131s
} 


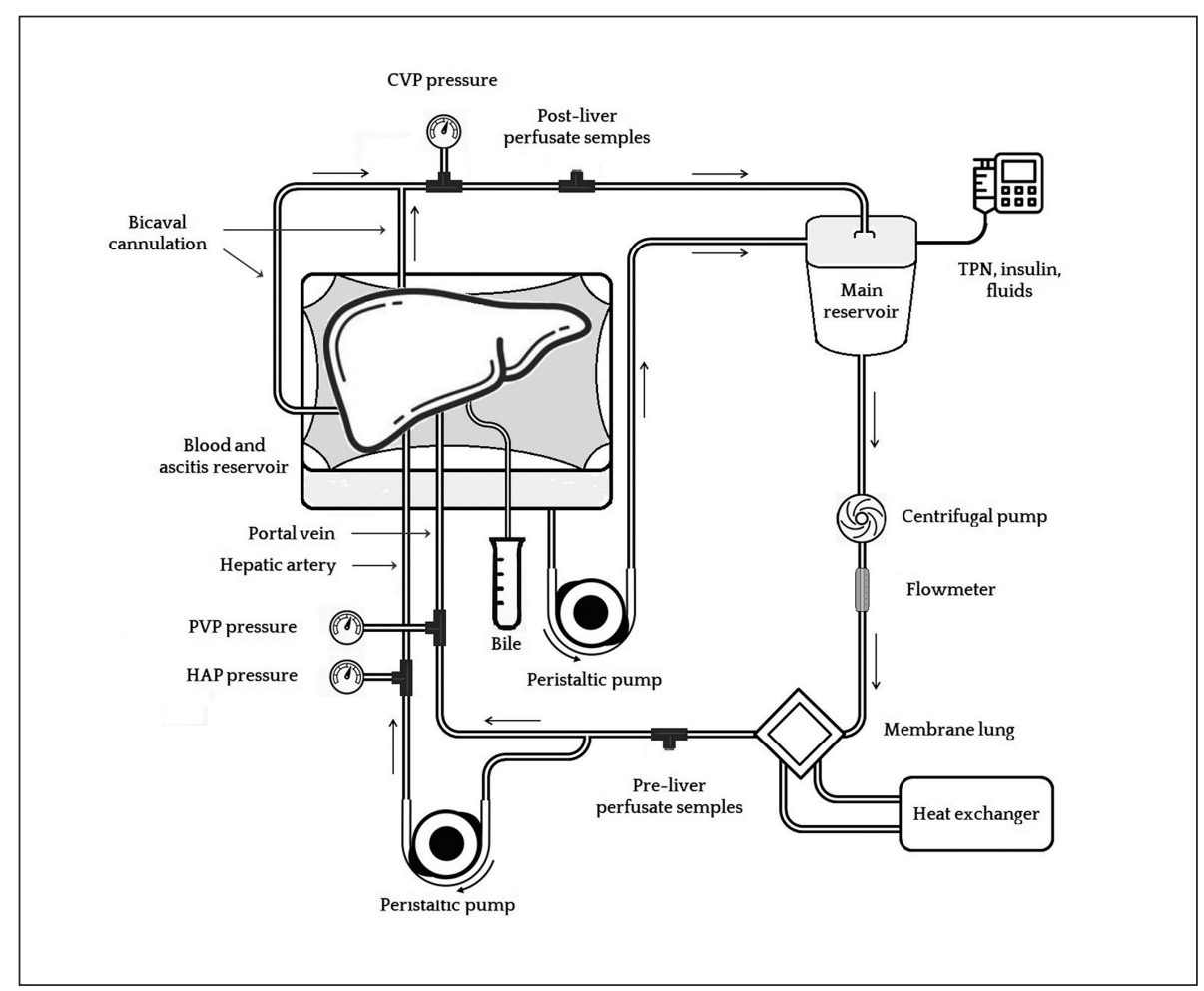

Fig. 1: Schematic representation of the normothermic machine perfusion system used in our experiments Closed circuit with cannulated inferior and superior vena cava and portal/ hepatic artery perfusion.

$\mathrm{O}_{2}$ content of post-liver perfusate $\left(\mathrm{C}_{\mathrm{POST}} \mathrm{O}_{2}\right)=[1.34 \times \mathrm{Hb}(\mathrm{g} / \mathrm{dL})$ POST-LIVER $\left.\times \mathrm{HbO}_{2}(\%)\right]+0.003 \times \mathrm{P}_{\text {POST-LIVER }}(\mathrm{mmHg})$

$\mathrm{C}_{\mathrm{PRE}} \mathrm{O}_{2}-\mathrm{C}_{\mathrm{POST}} \mathrm{O}_{2}=\triangle P R E-P O S T$

$\Delta_{\text {PRE-POST }} \times$ pump flow $(\mathrm{mL} / \mathrm{min}) \times 10=\dot{V^{\prime}}$

$\mathrm{C}_{\mathrm{PRE}} \mathrm{O}_{2} \times$ pump flow $(\mathrm{mL} / \mathrm{min}) \times 10=\mathrm{DO}_{2}$

Viability assessment of the liver

Graft viability was assessed after 180 min of the evaluation phase based on currently used clinical viability criteria (Mergental et al., 2016). The main criteria were: lactate level $<2.5 \mathrm{mmol} / \mathrm{L}$ and active bile production. Minor criteria were: perfusate $\mathrm{pH}>7.30$, arterial flow $>150 \mathrm{~mL}$, portal venous flow $>500 \mathrm{~mL}$ per min, and homogeneous graft perfusion with soft consistency. Grafts were classified as viable/transplantable (LT-G) or non-viable/non-transplantable grafts (nLT-G) according to these parameters.

\section{Evaluation of liver metabolism}

Samples were taken from the hepatic artery, portal and cava vein every $15 \mathrm{~min}$ for the first $60 \mathrm{~min}$ and every $30 \mathrm{~min}$ thereafter. Perfusion fluid samples were immediately analyzed to assess acid-base metabolism. $\mathrm{pH}$, partial pressure of oxygen $\left(\mathrm{pO}_{2}\right)$, partial pressure of carbon dioxide $\left(\mathrm{pCOO}_{2}\right), \mathrm{HCO}_{3}$-, base excess, glucose, lactate, $\mathrm{Na}^{+}, \mathrm{K}^{+}$, and $\mathrm{Cl}^{-}$levels were recorded. At baseline and every $60 \mathrm{~min}$, alanine aminotransaminase (ALT) and lactic dehydrogenases (LDH) were measured as markers of hepatocellular injury and alkaline phosphatases (ALP) to assess cholangiocyte injury. Lactate, glucose and potassium absolute concentration and release ratio $\left[\left(\mathrm{C}_{\text {start }}-\mathrm{C}_{\mathrm{end}}\right) / \mathrm{C}_{\text {start }}\right]$ were used as markers of graft metabolic recovery.

Sodium citrate was added to blood at the time of collection to obtain adequate anticoagulation by taking advantage of its ability to chelate calcium. Sodium citrate is mainly metabolized by the liver in zone 3 (Quistrorff et al., 1992; Kramer et al., 2003). There is a direct correlation between calcium release and metabolic activity. Therefore, we evaluated liver metabolic ability based on ionized calcium (i-Ca) concentration.

Indocyanine green dye (ICG) clearance test is clinically used to assess liver function before liver surgery. ICG is cleared by hepatocytes and secreted into the bile by cholangiocytes. For this reason, we decided to use ICG clearance to assess metabolism and secretion function of hepatocytes and cholangiocytes, respectively. Because this parameter was never before tested during NMP, we used it only in LT-G in order to obtain reference values. ICG dose was calculated as $0.5 \mathrm{mg} / \mathrm{kg}$ (pig weight). Perfusion fluid samples were collected before ICG administration and 1, 2, 5, 10, 15 and 30 min after. Bile samples $(\mathrm{n}=3)$ were analyzed after 15 and 30 min. 805-nm spectrophotometry was used to analyze the samples. Baseline values of samples collected before ICG administration were subtracted.

\section{Histology}

Tissue biopsies were performed 1) at the end of back table preparation, 2) at the beginning of NMP, and 3) at the end of NMP (240 $\mathrm{min})$. The samples were fixed in 4\% formalin or de-hydrated to assess wet-to-dry ratio (W/D). Formalin-fixed paraffin-embedded samples were stained with hematoxylin-eosin, Masson's trichrome, and reticulin histochemical staining. CD31 immuno- 
histochemical staining using DAKO automated system (Monoclonal Mouse Anti-human CD31 Endothelial Cell, Cole JC70A, Dako Omins) was used to evaluate endothelial integrity. 30 random fields per slide were investigated to determine the necrosis area.

To further reduce the number of animals used, we elected to use a histopathological outcome instead of performing a liver transplant. The histological samples were scored according to Brockmann et al. (2009), who demonstrated concordance among NMP results, histopathological analyses, and liver viability.

To determine wet-to-dry ratio (W/D), all the specimens were weighed with an analytical balance and dried in an oven at $50^{\circ} \mathrm{C}$ for $48 \mathrm{~h}$ (Dondossola et al., 2017b). W/D was calculated and used as an index of tissue edema. Liver specimens from other experiments (healthy livers with similar age) were used as controls for W/D ratio $(n=7)$.

\section{Statistical analysis}

All results are presented as mean \pm standard error of the mean (SEM). Statistical analysis was performed using Mann-Whitney test or one-way analysis of variance (ANOVA) for repeated measures, followed by Tukey's multiple comparison test to evaluate differences at each time point. $p<0.05$ was considered significant. Data were analyzed using SPSS Statistics 25 software (SPSS, Inc., Chicago, US).

\section{Results}

During the slaughterhouse procedures, 8 thoraco-abdominal blocks were successfully procured. One liver did not undergo NMP because of poor macroscopic appearance after cold flush (large non-perfused area and irregular appearance). The ex vivo procedure was performed in 7 livers. Liver surgical outcomes and functional parameters are the subject of the present research and are described in detail in this article. Together with liver grafts, lungs were procured and were subjected to successful ex vivo perfusion described elsewhere. Detailed data on procurement and perfusion techniques are not the focus of this publication, however, surgical details are given to fully describe the surgical technique. All materials, instruments, and manufacturers are listed in Tables S1 and $\mathrm{S} 2{ }^{1}$.

\subsection{Surgical procedure}

\section{En-bloc procurement}

The procurement procedure was performed within the slaughterhouse facility in clean (but not sterile) conditions, while perfusion solution, tubing, and drugs were kept sterile (Tab. S1, S2 ${ }^{1}$ ). The procurement took place during slaughterhouse work and without interfering with food production. In the first four cases, we adopted the surgical technique incompletely described by Grosse-Siestrup et al. (2001). The technique was subsequently modified owing to the poor graft viability during ex-situ perfusion. Indeed, optimization of the experimental protocol was the aim of this study.

Briefly, after exsanguination and immersion in hot water, a midline thoraco-laparotomy was performed, and the thoracic/abdominal organs were procured en-bloc by a slaughterhouse technician. Special attention was paid to avoid injuries of the liver parenchy- ma and of the inferior lung lobe during sternotomy and phrenectomy. The thoraco-abdominal block was then laid in a box containing $30^{\circ} \mathrm{C}$ saline solution (in the first four cases we used $4^{\circ} \mathrm{C}$ saline solution); the trachea was cannulated and manual ventilation was started $\left(\mathrm{FiO}_{2} 21 \%\right)$. Thoracic organs were separated from the abdominal organs by section of the inferior pulmonary ligaments, esophagus (after its ligation cranially to the diaphragm), aorta, and cava vein. The thoracic and abdominal blocks were placed separately in two different boxes $\left(30^{\circ} \mathrm{C}\right.$ saline solution; in the first four cases we used $4^{\circ} \mathrm{C}$ saline solution). The trachea was left as long as possible to secure correct ventilation (the right upper bronchus has a tracheal bronchus). Moreover, care was taken to retrieve the aorta without damage to its posterior partition to avoid injuries to the celiac and renal arteries. The thoracic organs were then cold flushed and used for separate experiments described elsewhere.

\section{Liver cold perfusion}

At the outset, the abdominal block was inspected. The aorta was identified and the celiac trunk was accessed through section of the aorta's posterior wall. A $12 \mathrm{Ch}$ urinary catheter was placed in the celiac trunk (secured with a 0 Polysorb tie placed at the origin of the celiac trunk) and a cold flush $\left(4^{\circ} \mathrm{C}\right)$ was started $(4 \mathrm{~L}$ of dialysis solution followed by $3 \mathrm{~L}$ of Celsior). Ice was progressively added to the $30^{\circ} \mathrm{C}$ saline solution boxes to progressively reduce the temperature to $4^{\circ} \mathrm{C}$.

During cold flush, the liver was dissected from the other abdominal organs. The first step consisted of access to the portal vein through dissection of the hepato-duodenal ligament (at the upper border of the pancreas) and section of the pancreas. The portal vein was isolated for $1 \mathrm{~cm}$. A $14 \mathrm{Ch}$ urinary catheter was inserted, and the catheter balloon was inflated $(5 \mathrm{~mL})$ to enable portal perfusion ( $1 \mathrm{~L}$ of Celsior). The portal vein dissection was done with particular care and as distal as possible to avoid injuries to the origin of the common hepatic artery. The surgical dissection was then completed with the detachment of gut, stomach, spleen, left kidney and right kidney. Careful attention was paid to a possible twist of the celiac pedicle due to the en-bloc procurement, which could result in injuries of the celiac trunk and hepatic artery. The main celiac and mesenteric vessels were ligated to avoid spillage of perfusion fluid. It was found best not to dissect the hepatic artery in this phase due to its small caliber. The bile duct was flushed at the slaughterhouse $(500 \mathrm{cc})$, but the gallbladder was not opened to avoid bleeding during NMP.

Cold perfusion was performed as soon as possible to minimize WIT. We recommend that if WIT is prolonged for experimental purposes, it should be done inside the animal body.

Maintaining a further $1000 \mathrm{cc}$ of cold $\left(4^{\circ} \mathrm{C}\right)$ Celsior infusion, the graft was placed in a plastic bag. At the end of perfusion, the graft was placed in an ice box and transferred to our preclinical facility.

\section{Surgical outcome}

The WIT (from death induction to cold perfusion) was $29 \pm 7 \mathrm{~min}$. Liver dissection lasted $20 \pm 3 \mathrm{~min}$. The time from death of the animal to ice storage of the liver graft was $63 \pm 10 \mathrm{~min}$. No major surgical damage was registered during ex vivo dissection. However, we have no data on the number of grafts discarded due to butcher damage during en-bloc procurement (a median of 3 graft/day 
were inspected to obtain an adequate organ). Liver graft weight was $1050 \pm 104 \mathrm{~g}$.

\section{Blood collection}

Blood was collected from the same animal from which the graft was procured during exsanguination (autologous blood). To avoid clotting, a closed collecting system was connected to a $2 \mathrm{~L} \mathrm{PVC}$ bag containing heparin $(25,000 \mathrm{UI})$ and sodium-citrate $(30 \mathrm{~mL})$. Blood contamination was reduced by adding ceftriaxone $1 \mathrm{~g}$. Blood bags were stored and transported at $24^{\circ} \mathrm{C}$. After arrival at the preclinical facility, the blood was separated by precipitation and the concentrated red blood cells were transferred to smaller blood bags through a $40 \mu \mathrm{m}$ filter. The total amount of blood collected per animal was $1450 \pm 200 \mathrm{~mL}$.

\section{Back table preparation}

Before ex-situ graft perfusion, back table preparation was performed to prepare liver vessels for cannulation. Small veins and arteries were tied to avoid spillage during perfusion. Cannulas were inserted and $1 \mathrm{~L}$ of Ringer lactate was infused through the hepatic artery. The bile duct was washed with $500 \mathrm{~mL}$ of Ringer lactate $\left(4^{\circ} \mathrm{C}\right)$ and a $14 \mathrm{G}$ venous cannula was placed in the gallbladder after closure of the cystic duct.

\subsection{Ex vivo dynamic perfusion}

\section{Perfusate composition}

The liver perfusion system consisted of a cellular perfusion fluid as described by Op Den Dries et al. (2013). The solution was freshly prepared in sterile conditions (Tab. S2 ${ }^{1}$ ). Total perfusate volume was $2370 \pm 340 \mathrm{~mL}$ with a hemoglobin concentration of $7.6 \pm 1.4 \mathrm{~g} / \mathrm{dL}$. To prevent bacterial contamination, $2 \mathrm{~g}$ ceftriaxone and $500 \mathrm{mg}$ metronidazole were added to the perfusate. No corticosteroids or other anti-inflammatory drugs were used.

\section{Liver perfusion system}

The liver graft was placed onto an organ chamber modified to let the dorsal liver lie on a modelled ad hoc perforated surface. The portal vein and the hepatic artery were connected to the circuit, together with the superior and inferior vena cava (closed circuit, Fig. 1 ). The perfusate was oxygenated by a membrane lung. The gas flow was titrated to maintain a normal $\mathrm{pH}$, starting with a sweep gas to blood flow ratio of 1:2 to avoid hypocapnic alkalosis during the initial warming period due to low tissue $\mathrm{CO}_{2}$ production. Sweep gas $\mathrm{FiO}_{2}$ was titrated to keep the arterial saturation $\left(\mathrm{SaO}_{2}\right)$ above $92 \%$ and avoid hyperoxia. In most cases, $21 \% \mathrm{FiO}_{2}$ was sufficient; an increase in $\mathrm{FiO}_{2}$ was required during only 3 NMP. The chamber was closed with a PVC lid to maintain humidity and temperature. The organ temperature was recorded with a probe placed between liver lobes. In some preliminary experiments, the core graft temperature was measured and was found equal to the temperature observed between liver lobes.

In order to maintain a liver temperature of $38^{\circ} \mathrm{C}$, the heat-exchanger connected to the membrane lung had to be set to $40^{\circ} \mathrm{C}$. Correct priming of the perfusion system was essential to prevent air embolism. To recirculate ascites and perfusate leakage, a further reservoir was placed under the perforated surface and connected to a roller pump. The drained fluids were pumped directly into the main reservoir.

\section{Liver perfusion protocol}

After static cold storage for $235 \pm 47 \mathrm{~min}$, the liver graft was connected to the circuit when a $\mathrm{pH}$ of 7.3 was reached by electrolyte adjustment (i.e., $\mathrm{NaHCO}_{3}$ infusion). The overall duration of liver perfusion was $353 \pm 138 \mathrm{~min}$ and included two steps (Fig. 2). The first 60 min were called "rewarming" and consisted of a progressive increase in graft temperature, pressure, and flow. Initial PVP was $3 \mathrm{mmHg}$, HAP $25 \mathrm{mmHg}$, and heat exchanger temperature $30^{\circ} \mathrm{C}$. Pressures and temperature were raised in 6 steps during the $60 \mathrm{~min}$ of rewarming to reach target values. No electrolytes or $\mathrm{pH}$ adjustments were made during this phase. The following phase was called "evaluation". It started when all parameters reached $100 \%$ of the target values and ended with the final graft evaluation at $240 \mathrm{~min}$. During this phase, Clinimix N14G30 $100 \mathrm{~mL} / \mathrm{h}$ and insulin $100 \mathrm{U} / \mathrm{h}$ were continuously infused through the portal vein. Portal vein and hepatic artery resistances and flow are shown in Figure 3.

\subsection{Graft evaluation}

According to Mergental criteria (Mergental et al., 2016), 3 (43\%) grafts were classified as nLT-G and $4(67 \%)$ as LT-G after $240 \mathrm{~min}$ NMP. The first 3 grafts of our series were all classified as nLT-G (Tab. 1).

\section{Histology after NMP}

At the end of the cold storage, histological evaluation of hepatocyte and sinusoidal integrity was grossly normal without signs of inflammation, steatosis or fibrosis. CD31 was used to mark endo-

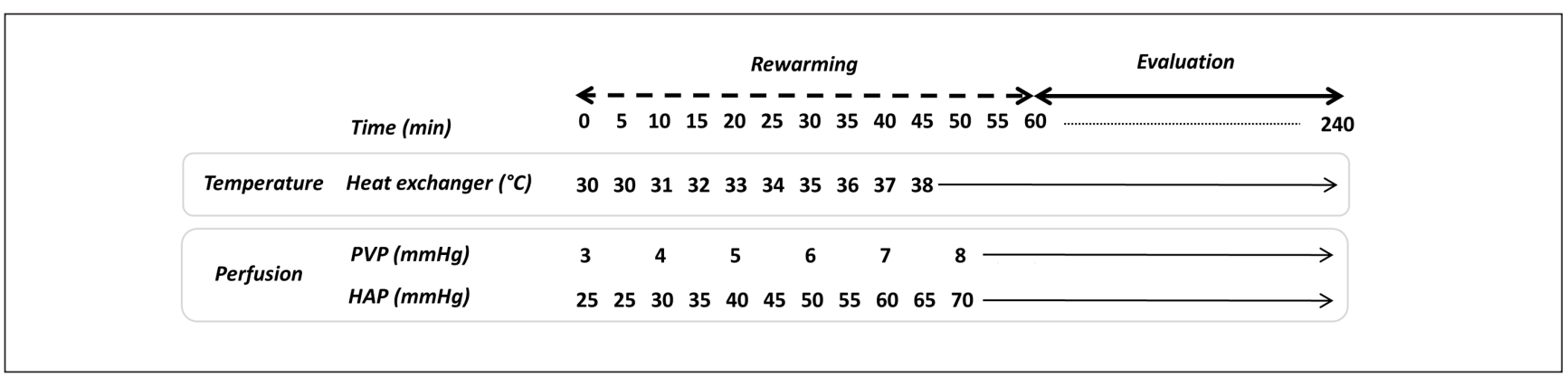

Fig. 2: Schematic overview of normothermic machine perfusion protocol

PVP, portal vein pressure; HAP, hepatic artery pressure 

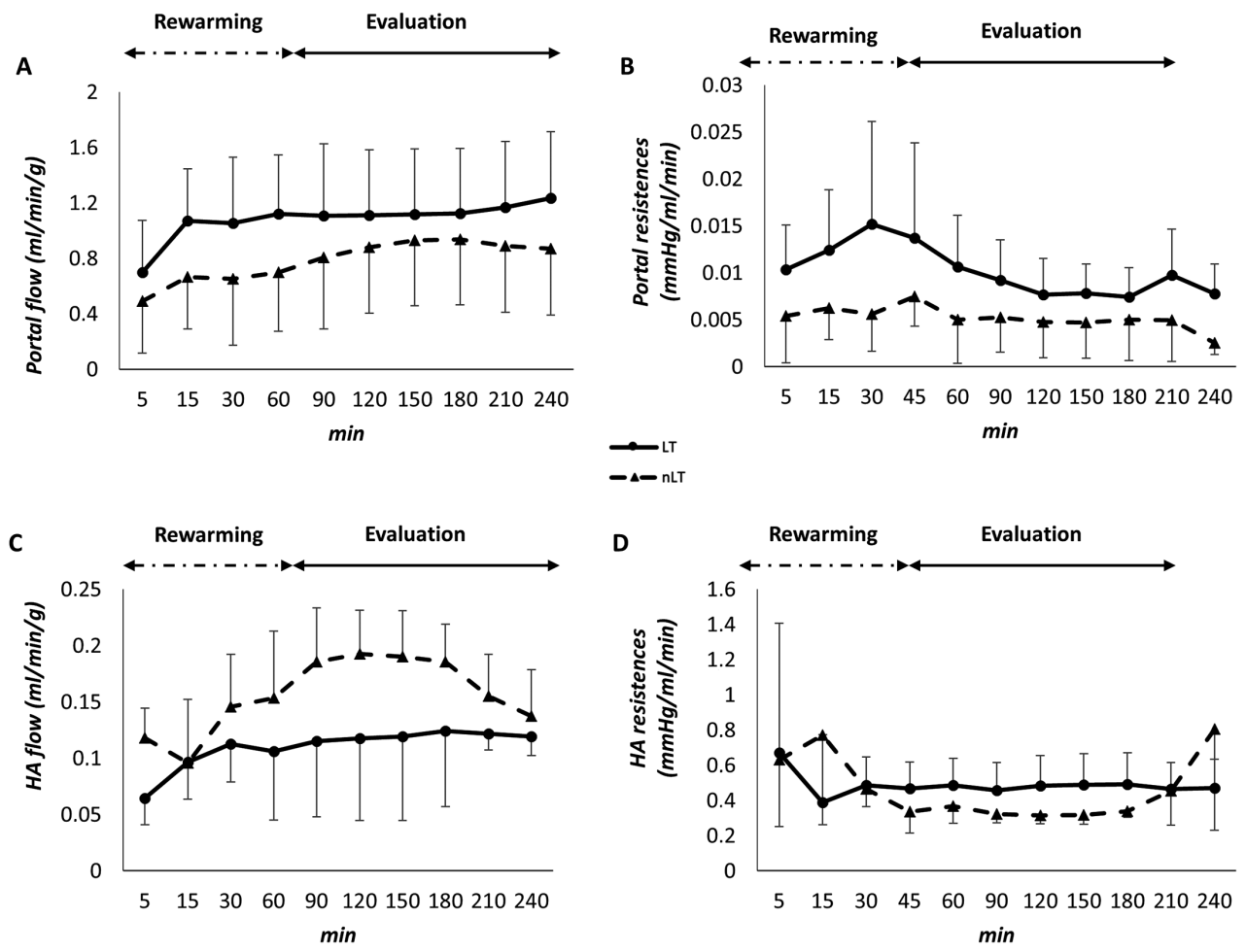

Fig. 3: Portal vein flow (A) and resistances (B) and hepatic artery flow (C) and resistances (D) during normothermic machine perfusion

$\mathrm{LT}, \mathrm{n}=4 ; \mathrm{nLT}, \mathrm{n}=3$. Data are expressed as mean \pm SEM. $\mathrm{HA}$, hepatic artery.

Tab. 1: Classification of the seven perfused grafts according to Mergental et al. (2016) criteria

\begin{tabular}{|c|c|c|c|c|c|c|c|}
\hline & Liver 1 & Liver 2 & Liver 3 & Liver 4 & Liver 5 & Liver 6 & Liver 7 \\
\hline \multicolumn{8}{|l|}{ Major criteria } \\
\hline Lactate $<2.5 \mathrm{mmol} / \mathrm{L}$ & no & no & no & yes & yes & no & yes \\
\hline Bile production & no & no & no & yes & yes & yes & yes \\
\hline Score & $0 / 2$ & $0 / 2$ & $0 / 2$ & $2 / 2$ & $2 / 2$ & $1 / 2$ & $2 / 2$ \\
\hline \multicolumn{8}{|l|}{ Minor criteria } \\
\hline $\mathrm{pH}>7.3$ & yes & yes & no & yes & yes & no & yes \\
\hline HA flow > $150 \mathrm{~mL}$ & yes & yes & yes & yes & yes & yes & no \\
\hline PV flow > $500 \mathrm{~mL}$ & yes & yes & yes & yes & yes & yes & yes \\
\hline Homogenous perfusion & no & yes & no & yes & yes & yes & yes \\
\hline Score & $3 / 4$ & $4 / 4$ & $2 / 4$ & $4 / 4$ & $4 / 4$ & $3 / 4$ & $3 / 4$ \\
\hline Classification & $\mathrm{nLT}$ & $\mathrm{nLT}$ & $\mathrm{nLT}$ & $\mathbf{L T}$ & LT & LT & LT \\
\hline
\end{tabular}

thelial cells and evaluate endothelial and sinusoidal integrity. Conversely, during NMP, a progressive deterioration of hepatocellular and sinusoidal architecture was observed in nLT-G $(\mathrm{p}=0.042)$, while only few changes were observed in LT-G (Fig. 4). Although a clear inflammatory response arose during perfusion in both groups, the diffused hepatocellular necrosis of nLT-G resulted in a marked neutrophil recruitment or proliferation. Similarly, biopsies from nLT-G showed diffuse small droplet steatosis deposition (from $10 \%$ to $50 \%$ ). These observations could be considered a para-physiological attempt of liver grafts to deal with hepatocyte and sinu- 


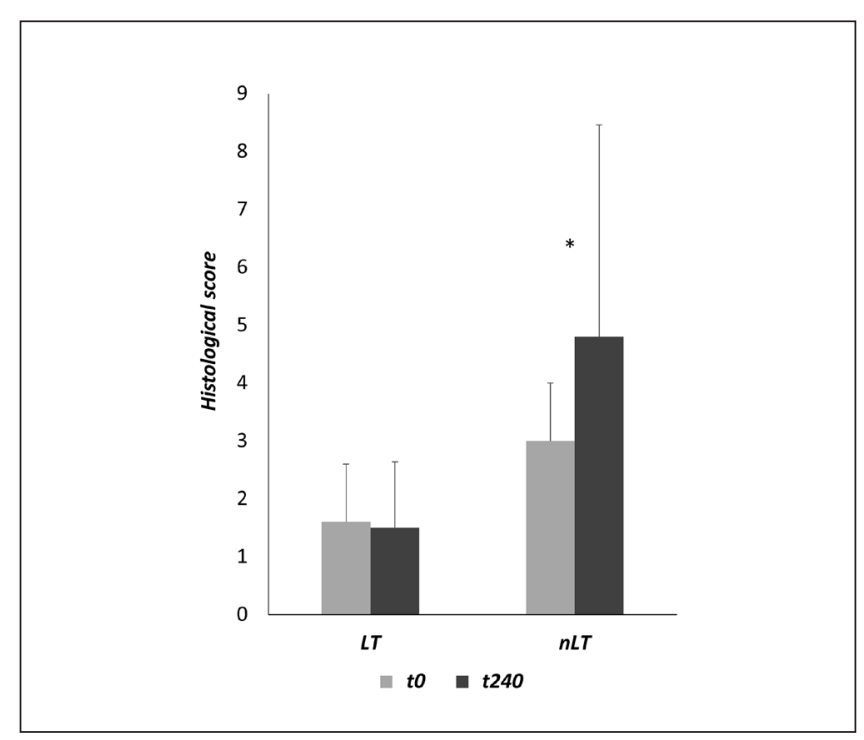

Fig. 4: Histologic scoring of hemorrhage and necrosis of LT-G and nLT-G

Histologic findings were evaluated at the indicated time points in the experimental groups. The severity of these changes was evaluated by the scoring system validated by Brockman et al. (2009). $L T, n=4 ; n L T, n=3$. Data are expressed as mean $\pm S E M$ $\left({ }^{*}, p=0.042\right)$.

soidal damage. Indeed, microvesicular steatosis was probably the result of deficient liver metabolism, and immune cell infiltrate was likely secondary to increased chemotactic signals.

As hepatocyte injury can lead to graft swelling, W/D ratio was assessed. Although W/D ratio in nLT-G tended to be higher at the end of the evaluation phase, the difference was not statistically significant (LT-G $3.013 \pm 0.126$ versus $n L T-G 3.221 \pm 0.181, \mathrm{p}=$ $0.069)$. The W/D ratio in the 7 control livers, which had not undergone NMP, was $2.975 \pm 0.143$, significantly lower than in nLT-G (p $=0.038$ ).

Ex-situ metabolic evaluation of liver grafts

The main biomarkers measured during NMP are shown in Table 2.

During the rewarming phase (from 0 to $60 \mathrm{~min}$ ), glucose concentration in the perfusate increased in both LT-G and nLT-G $(\mathrm{p}=0.001)$. Glucose release ratio was $-0.54 \pm 0.18$ in LT-G and $0.08 \pm 0.11$ in nLT-G $(\mathrm{p}=0.03)$ (Fig. 5B). Lactate concentration, measured in the cold flush at the end of back table, was $4.3 \pm 2.6 \mathrm{mmol} / \mathrm{L}$ in LT-G and $4.2 \pm 4.1 \mathrm{mmol} / \mathrm{L}$ in $\mathrm{nLT}-\mathrm{G}$ $(\mathrm{p}=0.626)$. Furthermore, the lactate concentration in the perfusion fluid before liver graft connection to NMP was $8.9 \pm 2.7 \mathrm{mmol} / \mathrm{L}$ in LT-G and $3.7 \pm 0.5$ in nLT-G $(p=0.061)$. Even though LT-G lactate levels at the beginning of NMP were increased, there was no significant difference in lactate concentration between the two groups $(\mathrm{p}=0.224)$. Conversely, lactate concentration significantly decreased in LT-G, while it remained stable in nLT-G $(\mathrm{p}=0.02)$ (Fig. 5A). Lactate release ratio was $0.55 \pm 0.26$ in LT-G vs $-0.17 \pm 0.64$ in $n L T-G(p=0.022)$. Potassium concentration in the perfusion fluid was consistently higher in $\mathrm{nLT}-\mathrm{G}$ relative to LT-G $(p=0.020)$. LT-G had a higher potassium release ratio compared

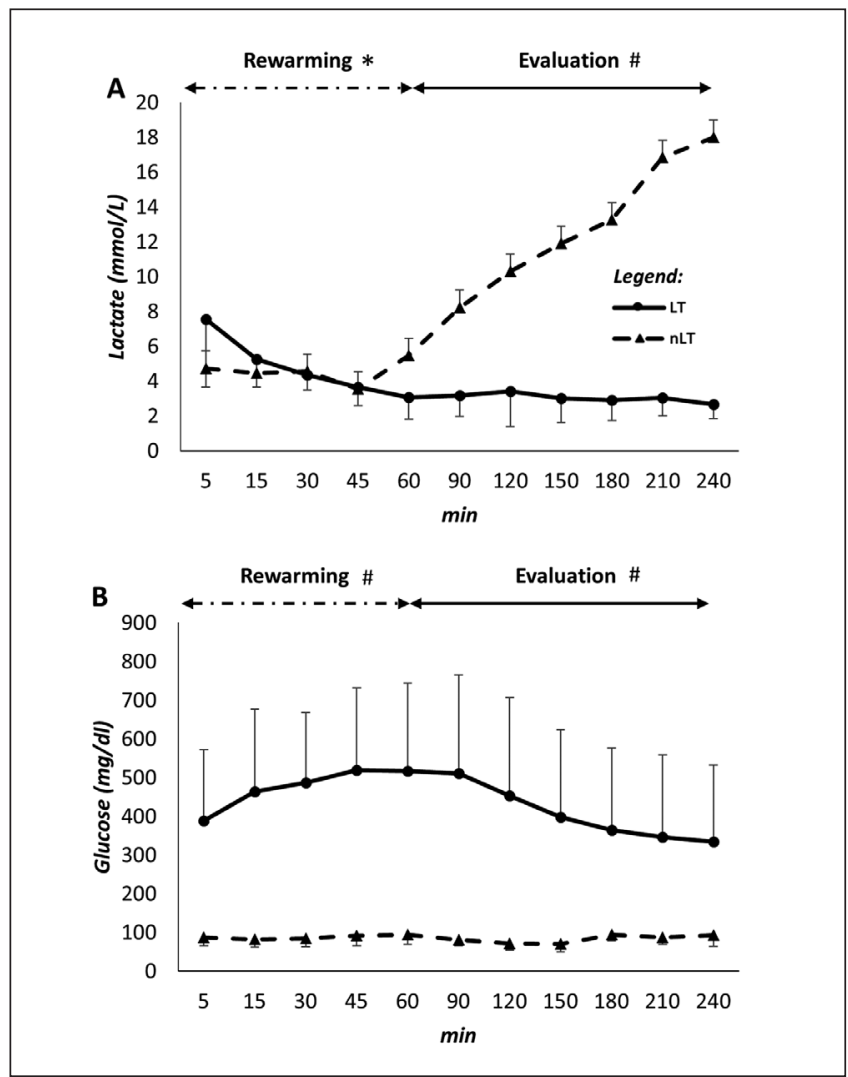

Fig. 5: Lactate (A) and glucose (B) concentrations in the two study groups during rewarming and evaluation phase of normothermic machine perfusion

$\mathrm{LT}, \mathrm{n}=4 ; \mathrm{nLT}, \mathrm{n}=3$. Data are expressed as mean \pm SEM

$\left({ }^{*}, p=0.02 ; \#, p<0.001\right)$.

to $\mathrm{nLT}-\mathrm{G}(-0.46 \pm 0.22$ in LT-G vs $0.17 \pm 0.64$ in $\mathrm{nLT}-\mathrm{G}, \mathrm{p}=0.047)$. Interestingly, the potassium and lactate release ratios showed a significant linear correlation $\left(\mathrm{R}^{2}=0.570 ; \mathrm{p}=0.05\right)$ (Fig. $\left.\mathrm{S}^{1}{ }^{1}\right) . \dot{V O} \mathrm{O}_{2}$ increased over time in LT-G, while it decreased in $\mathrm{nLT}$ after an early burst ( $\mathrm{p}=0.001)$ (Fig. 6).

During the evaluation phase, baseline AST and LDH levels were higher in nLT-G compared to LT-G (AST: 14'962 $\pm 1076 \mathrm{U} / 1$ vs $1305 \pm 1192 \mathrm{U} / 1, \mathrm{p}=0.001$; LDH: 10 ' $170 \pm 540$ vs $1584 \pm 946 \mathrm{U} / 1, \mathrm{p}<0.001)$. During machine perfusion, hepatocellular damage (AST and LDH) significantly increased in nLT-G (endNMP: AST 22’315 \pm 3560 U/l; LDH 14'237 \pm 1617 U/1), while it was stable in LT-G (end-NMP: AST $1444 \pm 800$ U/l; LDH 1674 \pm 1707 U/L) ( $p<0.001)$ (Fig. S2 $\left.{ }^{1}\right)$. Consistently, NMP preserved cholangiocyte function in LT-G, while a progressive bile duct injury was observed in LT-G $(p=0.001)$ (Fig. S3 $\left.{ }^{1}\right)$. Bile production was almost absent in the 3 nLT-G and in 1 LT-G. The mean bile volume/h was $8 \pm 5 \mathrm{~mL} / \mathrm{h}$. Lactate remained stable in LT-G (t60 min: $3.1 \pm 1.3 \mathrm{mmol} / \mathrm{L}$; t2 $40 \mathrm{~min}: 2.7 \pm 0.8 \mathrm{mmol} / \mathrm{L}$ ), whereas it increased in LT-G to more than $300 \%$ (t60 min: $5.4 \pm 3.5 \mathrm{mmol} / \mathrm{L}$; t240 min: $18.0 \pm 2.0)(\mathrm{p}<0.001)$ (Fig. 5A). Lactate release ratio was $0.073 \pm 0.310$ in LT-G vs $-3.749 \pm 3.811$ in nLT-G $(\mathrm{p}<0.001)$. Glucose concentration decreased in LT-G, while it remained stable in $\mathrm{nLT}-\mathrm{G}(\mathrm{p}=0.001)$ (Fig. 5B). Glucose release ratio was 0.384 


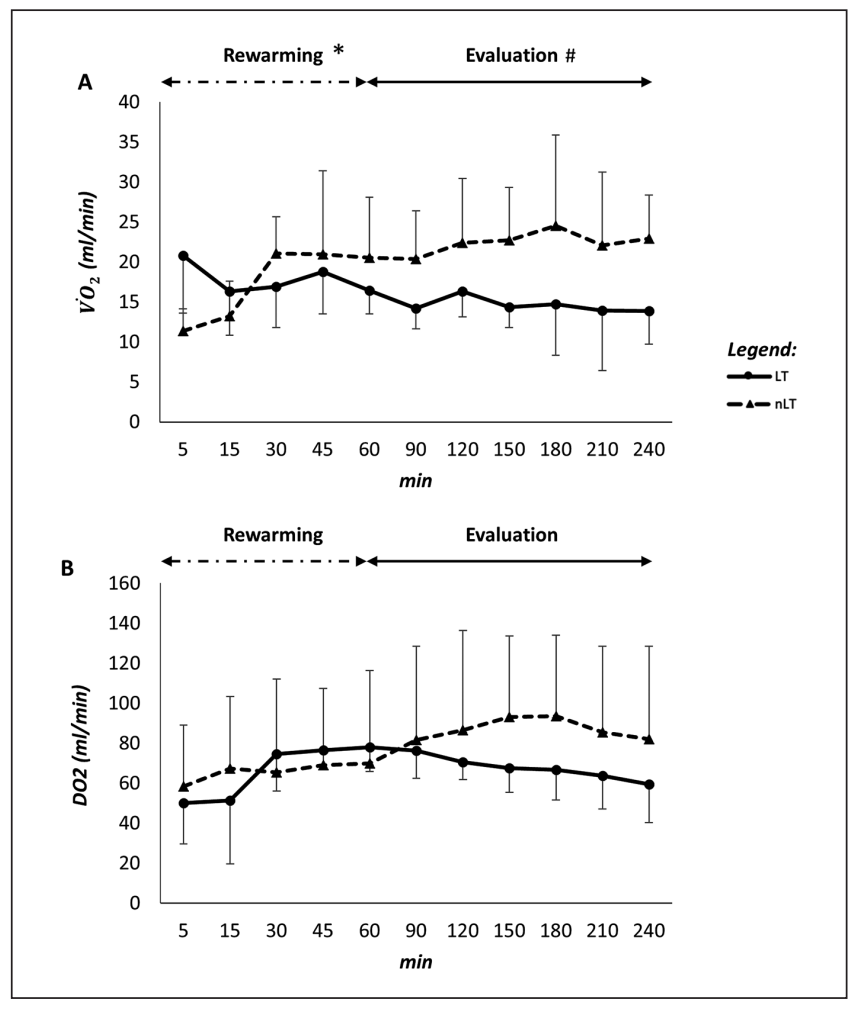

Fig. 6: Oxygen metabolic parameters during normothermic machine perfusion in the two study groups

(A) Oxygen consumption $\left(\mathrm{VO}_{2}\right)$ ( ${ }^{*}, p=0.001$ and \#, $\left.p<0.001\right)$.

(B) Oxygen delivery $\left(\mathrm{DO}_{2}\right)$. $\mathrm{LT}, \mathrm{n}=4 ; \mathrm{nLT}, \mathrm{n}=3$. Data expressed as mean \pm SEM.

\pm 0.108 in LT-G vs $0.048 \pm 0.429$ in nLT-G. A stable i-Ca was observed in nLT-G, whereas it increased in LT-G groups $(p=0.005)$ (i-Ca release ratio: LT-G $-1.355 \pm 0.582$ vs $n L T-G-0.107 \pm 0.231$, $\mathrm{p}=0.018)$. Hemoglobin concentration decreased to $2.3 \pm 0.9 \mathrm{~g} / \mathrm{dL}$ in $\mathrm{n}-\mathrm{LT}-\mathrm{G}$, while it remained stable in LT-G $(0.6 \pm 0.9 \mathrm{~g} / \mathrm{dl})(\mathrm{p}=$ 0.009) (Fig. S4 ${ }^{1}$ ). $\mathrm{VO}_{2}$ decreased in nLT-G, while it increased in LT-G $(\mathrm{p}<0.001)$. Transported oxygen $\left(\mathrm{DO}_{2}\right)$ was not different in the two groups $(\mathrm{p}=0.764)$ (Fig. 6).

In the 4 LT-G, $25 \pm 3 \mathrm{mg}$ of ICG were infused. R15 was $5.4 \pm 1.1 \%$ in the perfusate and bile concentration of ICG was $131 \pm 18 \%$ at $15 \mathrm{~min}$ and $2049 \pm 188 \%$ at $30 \mathrm{~min}$ (Fig. 7).

\section{Discussion}

The present research shows that organs retrieved from a slaughterhouse can be used as a model of liver DCD donation. Procurement and ex-situ perfusion procedures were optimized and accurately described in order to achieve an NMP model that resembles the clinical scenario. The results should provide significant information to develop effective preclinical research while complying with the 3Rs.

DCD donors are considered a valuable potential resource to increase the number of organs suitable for transplantation (Manyalich et al., 2018). However, the use of liver grafts derived from

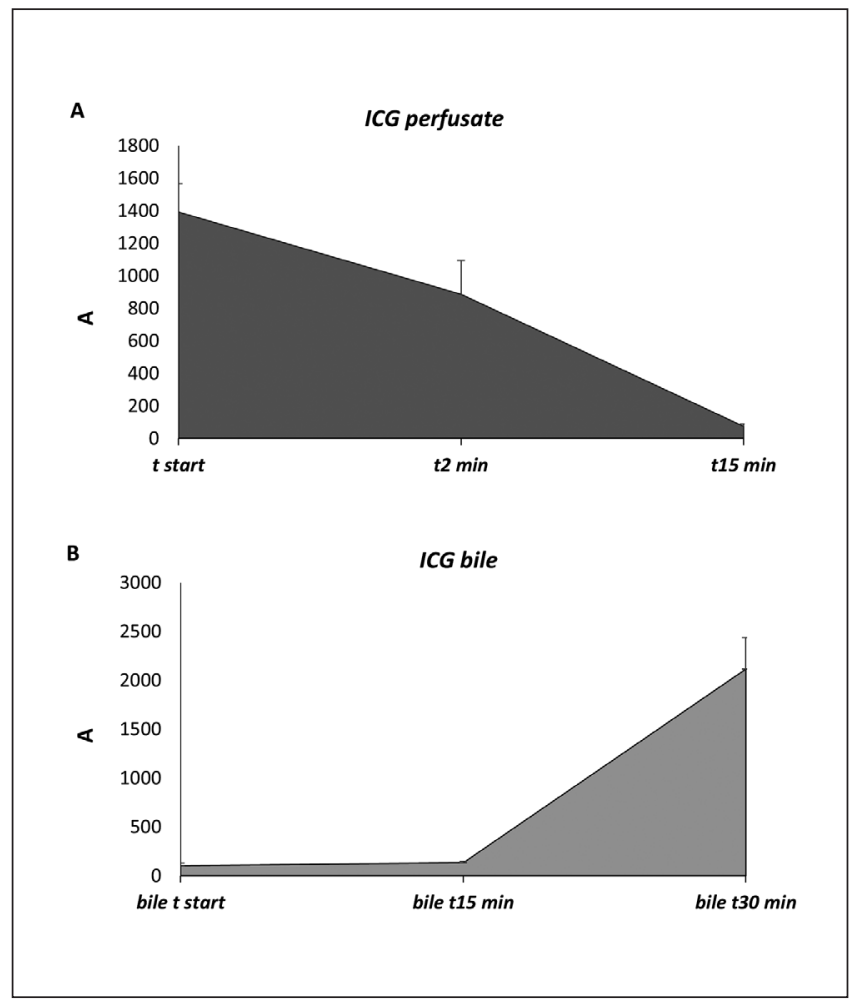

Fig. 7: Indocyanine green dye (ICG) clearance in perfusate (A) and secretion in bile (B) during normothermic machine perfusion in LT-G

Bile secretion was evaluated only in the 3 (of 4) LT grafts that produced bile.

these donors is burdened by sub-optimal post-LT outcome (Foley et al., 2011). Although several detrimental factors contributing to this result have been identified (e.g., WIT), the underlying biomolecular mechanisms are still largely unknown. Undoubtedly, these detrimental factors need to be extensively investigated and repaired by committed research.

Recently introduced evaluation and reconditioning strategies have demonstrated their ability to revert the detrimental impact of WIT and IRI (Dutkowski et al., 2015; Hessheimer and Fondevila, 2017). In our experience, machine perfusion was identified as a valuable technology to recondition ECD and DCD liver grafts (Dondossola et al., 2018). Machine perfusion is an emerging approach with numerous technical and biological aspects presently being explored, adapted and improved. However, reactions elicited during ex-situ dynamic perfusion and identification of criteria to establish liver graft viability are still ambiguous and their definition appears mandatory (Dutkowski et al., 2019).

To this purpose, based on low cost, reproducibility and better understanding of subcellular events, rat models have been widely used. However, these models provide results that have a low translational impact, requiring further experiments prior to a reliable clinical application (Bassani et al., 2016). Conversely, the translational potential of pig models is definitely greater. However, pig models have high costs in terms of life and economy, and non-experimental animals rather should be used for ethical reasons (Dan- 
Tab. 2: Main characteristics of normothermic machine perfusion during rewarming and evaluation phase * W/D was compared to native liver $(2.975 \pm 0.143, p=0.038)$. LT-G, transplantable grafts; $n L T-G$, non-transplantable grafts; n/a, not appliable

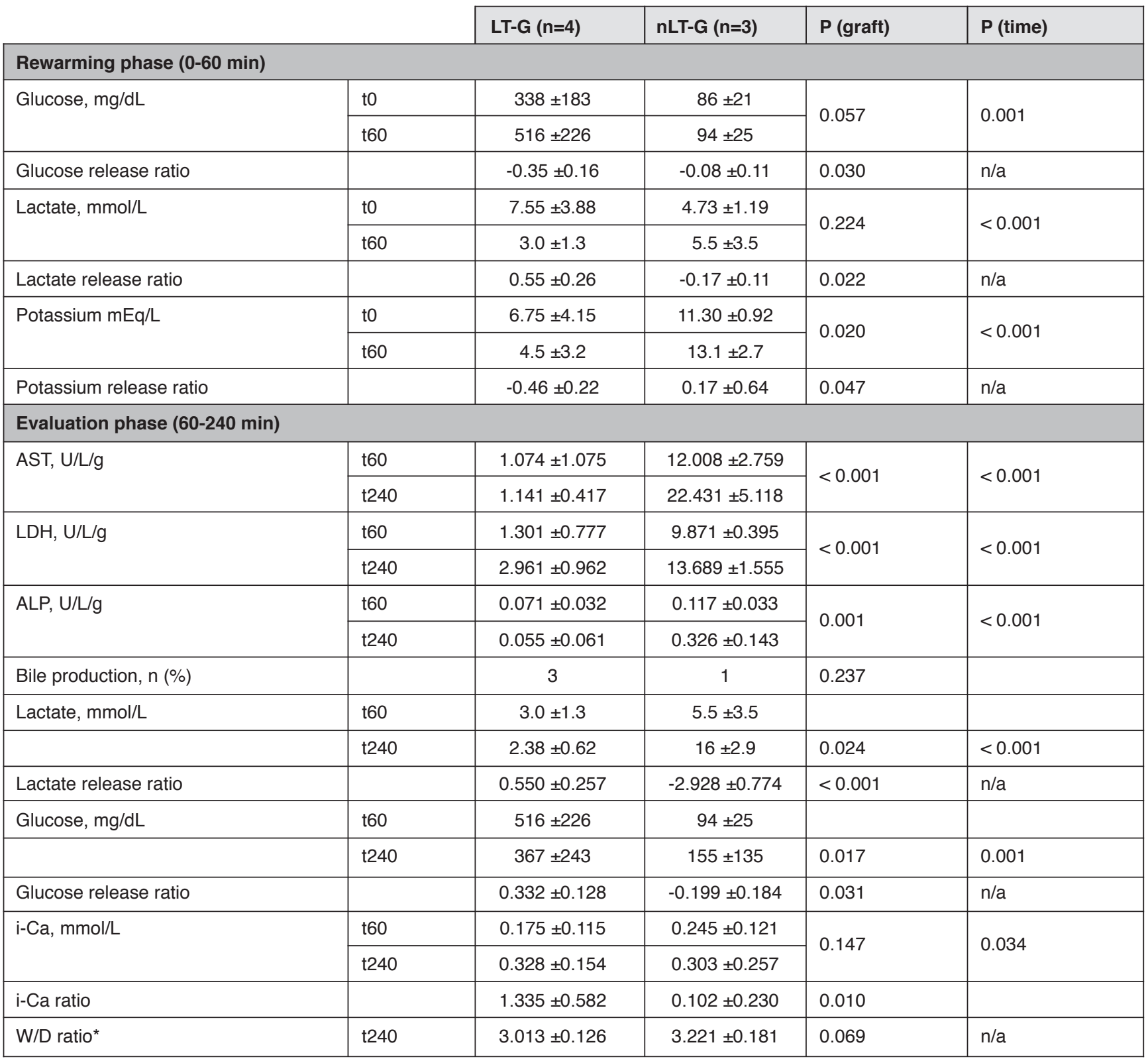

iel et al., 2018; Chung et al., 2019). Unfortunately, among the many studies on DCD and machine perfusion published over the last 20 years, only few have used non-dedicated experimental animals (Grosse-Siestrup et al., 2001, 2002a,b). This limitation is probably the result of incompletely standardized procurement and perfusion protocols of organs derived from slaughterhouse animals.

Although the use of commercial animals to study specific biomolecular phenomena has its challenges, the organs can be used to refine physiological and technical aspects of NMP in accordance with the 3 Rs. Furthermore, in our setting, the costs connected to slaughterhouse procurement were lower than for a model based on dedicated animals. For this reason, we focused our research on the optimization of all the steps required to standardize a slaughterhouse NMP model. The detailed description of our procurement and perfusion procedures can motivate researchers unfamiliar with the 3 Rs to try this approach and contribute to stimulate awareness of this topic with a substantial impact on animal use.

The porcine liver ex-situ perfusion model described in this paper was initially based on the technique described by GrosseSiestrup (2001). However, the first four grafts retrieved according to that procedure and perfused with a clinically fashioned circuit resulted in non-viable organs. Consequently, technical refinement of critical steps (organ procurement, blood collection, and perfusion system) was pursued. Particular attention was paid to organ 
Tab. 3: Main metabolic characteristics of transplantable (LT-G) and non-transplantable (nLT-G) grafts during the rewarming and evaluation phase

Rew., rewarming; Ev., evaluation; i-Ca, ionized calcium; $\mathrm{VO}_{2}$, oxygen consumption; $\mathrm{n} / \mathrm{a}$, not appliable

\begin{tabular}{|c|c|c|c|c|c|c|}
\hline & \multirow[t]{2}{*}{ Phase } & \multicolumn{5}{|c|}{ Viability criteria } \\
\hline & & Glucose & Lactate & Potassium & $\mathrm{i}-\mathrm{Ca}$ & $\mathrm{VO}_{2}$ \\
\hline \multirow{2}{*}{ LT-G } & Rew. (0-60 min) & released & uptake & uptake & $\mathrm{n} / \mathrm{a}$ & increased \\
\hline & Ev. $(60-240 \mathrm{~min})$ & uptake & uptake & $\mathrm{n} / \mathrm{a}$ & increase & increased \\
\hline \multirow{2}{*}{$n L T-G$} & Rew. (0-60 min) & stable & stable & released & $\mathrm{n} / \mathrm{a}$ & decreased \\
\hline & Ev. $(60-240 \mathrm{~min})$ & released & released & $\mathrm{n} / \mathrm{a}$ & stable & decreased \\
\hline
\end{tabular}

cooling. Indeed, excessively rapid graft cooling caused an increase in vascular resistance, leading to impaired organ perfusion (Tashiro et al., 2014). After protocol refinement, the temperature was progressively decreased, which led to a homogenous cold graft perfusion; adequate blood wash-out enabled reliable and efficient ex-si$t u$ normothermic perfusion.

In nLT-G grafts, ischemic areas during NMP represented a major problem that led to histological progression of liver damage. Conversely, no ischemic areas were observed in LT-G, perfusion parameters improved, and our results are comparable to those obtained in other preclinical research based on experimental animals and in clinical series (Ravikumar et al., 2016; Banan et al., 2015).

We used the criteria proposed by Mergental et al. (2016) to evaluate graft viability at the end of NMP. We considered whether the differences in weight and vascular flow between humans and pigs could affect our evaluation. Different studies have analyzed the in vivo pig splanchnic flow (Iozzo et al., 2007; Slimani et al., 2008; Winterdahl et al., 2011). According to the reported data and our mean graft weight, the estimated in vivo mean portal flow ranges from 1100 to $1500 \mathrm{~mL} / \mathrm{min}$ and hepatic artery flow from 150 to $250 \mathrm{~mL} / \mathrm{min}$. These values are fully comparable with human values, and the resulting graft classification showed full concordance with histopathological evaluation.

The Cambridge group (Watson et al., 2017) demonstrated a significant correlation between transaminase concentration in the perfusion fluid and post-LT graft function. Consistently, in the present study, nLT-G showed higher baseline AST and LDH levels that markedly increased during NMP. These changes matched the pronounced deterioration of hepatocytes shown by histopathological analysis. Therefore, a concordance between Mergental criteria and Cambridge observations could be shown for the first time. Further, the progressive increase in hepatocellular necrosis markers during NMP is a well-known phenomenon (Verhoeven et al., 2014) that underlines the need for novel strategies to limit ex-situ reperfusion damage, especially in severely damaged grafts (Boteon, 2019; de Vries et al., 2019).

Although the reconditioning and preservation capacity of NMP has been the focus of relevant publications, the metabolic profile of liver grafts during NMP has been investigated insufficiently to date. In particular, the role of the rewarming phase in understanding perfusion results has clearly been underestimated. To mend this limitation, an accurate metabolic evaluation of the NMP rewarming phase is provided in our study. The oxygen debt, accu- mulated during ischemia, is restored during reperfusion, and analysis of $\mathrm{O}_{2}$ metabolism can help the assessment of graft viability and evaluation of ischemic damage. Interestingly, in vivo studies on acute liver failure have directly related the $\mathrm{VO}_{2}$ to the remnant liver function (Clemmesen et al., 1999; Hart et al., 2003). A low $\mathrm{VO}_{2}$ could be seen as a marker of cell necrosis or dysfunction, causing increased lactate levels, inadequate glucose metabolism, and increased potassium concentration, likely due to cell death and $\mathrm{Na}^{+}-\mathrm{K}^{+}$-ATPase-dependent pump inactivation. Conversely, LT-G showed higher $\mathrm{VO}_{2}$, adequate lactate metabolism, and active glucose and potassium absorption. These data suggest that the extent of oxygen debt during warm/cold ischemia exceeds the nLT-G liver graft's ability to adequately repair it and to sustain the simultaneous metabolic demand of ex-situ rewarming (Bjerkvig et al., 2016). The subsequent microcirculation damage and mitochondrial dysfunction leads to cell necrosis and non-transplantability of the liver graft, as demonstrated by hepatocellular necrosis and lactate accumulation in nLT-G. The metabolic parameters of the rewarming phase in our research (glucose, lactate and potassium release ratio) are in agreement with the evaluation criteria provided by Mergental et al. (2016) (Tab. $3)$. This observation deserves particular emphasis as an early graft evaluation procedure could have considerable clinical and practical implications. Furthermore, in accordance with our recent publication on an NMP rat model (Dondossola et al., 2019), these metabolic parameters, together with citrate clearance, easily measured as i-Ca and ICG clearance, a well-known in vivo liver function test, could be a suitable parameter combination to precisely evaluate graft function and transplantability.

We are aware of potentially problematic issues in our model. First of all, an extended WIT could be common. Indeed, a very short WIT requires a well-programmed synergy with slaughterhouse technicians to reduce the interval between sacrifice and organ procurement. Further, we observed substantial hemolysis in nLT-G that remains unexplained. Of interest, hemolysis was a clinically relevant issue in other NMP series (Watson et al., 2017). Lactate levels in the perfusate before NMP graft connection tended to be higher in LT-G, which is probably due to blood composition and collection procedures that cannot be controlled. However, it should be considered that similar changes occur in clinical practice as well and do not appear to affect graft quality.

In conclusion, the present model with a prolonged no-flow period (WIT $>20 \mathrm{~min}$ ) shows that slaughterhouse organs can be used 
to study and recondition liver grafts after cardiocirculatory arrest. Indeed, liver grafts obtained from animals with peri-mortem events that resemble DCD donation demonstrated full viability at the end of the NMP evaluation. The optimized ex-situ perfusion system resembles the physiological in vivo response to ischemia-reperfusion injury and could be used to test graft viability as well as to study liver physiology and metabolism. These data represent a substantial advance in translational research that promotes a reduction of dedicated experimental animals.

\section{References}

Banan, B., Chung, H., Xiao, Z. et al. (2015). Normothermic extracorporeal liver perfusion for donation after cardiac death (DCD) livers. Surgery (United States) 158, 1642-1650. doi:10.1016/j.surg.2015.07.016

Banan, B., Watson, R., Xu, M. et al. (2016). Development of a normothermic extracorporeal liver perfusion system toward improving viability and function of human extended criteria donor livers. Liver Transpl 22, 979-993. doi:10.1002/1t.24451

Bassani, G. A., Lonati, C., Brambilla, D. et al. (2016). Ex vivo lung perfusion in the rat: Detailed procedure and videos. PLoS One 11, e0167898. doi:10.1371/journal.pone.0167898

Bjerkvig, C. K., Strandenes, G., Eliassen, H. S. et al. (2016). "Blood failure" time to view blood as an organ: How oxygen debt contributes to blood failure and its implications for remote damage control resuscitation. Transfusion 56, Suppl 2, S182-189. doi:10.1111/trf.13500

Blackstock, M. J. and Ray, D. C. (2014). Organ donation after circulatory death: An update. Eur J Emerg Med 21, 324-329. doi:10.1097/MEJ.0000000000000082

Boteon, Y. L. (2019). Machine perfusion of the liver: Which is the best technique to mitigate ischaemia-reperfusion injury? World J Transplant 9, 14-20. doi:10.5500/wjt.v9.i1.14

Brockmann, J., Reddy S., Coussios, C. et al. (2009). Normothermic perfusion: A new paradigm for organ preservation. Ann Surg 250, 1-6. doi:10.1097/SLA.0b013e3181a63c10

Chung, W. Y., Wanford, J. J., Kumar, R. et al. (2019). An ex vivo porcine spleen perfusion as a model of bacterial sepsis. ALTEX 3, 29-38. doi:10.14573/altex.1805131

Clemmesen, J. O., Gerbes, A. L., Gülberg, V. et al. (1999). Hepatic blood flow and splanchnic oxygen consumption in patients with liver failure. Effect of high-volume plasmapheresis. Hepatology 29, 347-355. doi:10.1002/hep.510290206

Daniel, C. R., Labens, R., Argyle, D. et al. (2018). Extracorporeal perfusion of isolated organs of large animals - Bridging the gap between in vitro and in vivo studies. ALTEX 35, 77-98. doi:10.14573/altex.1611291

De Vries, Y., Matton, A. P. M., Nijsten, M. W. N. et al. (2019). Pretransplant sequential hypo- and normothermic machine perfusion of suboptimal livers donated after circulatory death using a hemoglobin-based oxygen carrier perfusion solution. Am J Transplant 19, 1202-1211. doi:10.1111/ajt.15228

Dondossola, D., Cavenago, M., Antonelli, B. et al. (2017a). Sent liver grafts do not have a detrimental impact on post-transplant outcome. Transplant Proc 49, 1388-1393. doi:10.1016/j. transproceed.2017.03.084
Dondossola, D., Lonati, C. and Rossi, G. (2017b). Ex-vivo tissue determination of water fraction in associating liver partition with portal vein ligation for staged hepatectomy. Surgery (United States). doi:10.1016/j.surg.2017.10.040

Dondossola, D., Lonati, C., Zanella, A. et al. (2018). Preliminary experience on hypothermic oxygenated machine perfusion in an Italian liver transplant center. Transplant Proc 51, 111-116. doi:10.1002/lt.25315

Dondossola, D., Santini, A., Lonati, C. et al. (2019). Human red blood cells as oxygen carriers to improve ex-situ liver perfusion in a rat model. J Clin Med 8, E1918. doi:10.3390/ jcm8111918

Dutkowski, P., Polak, W., Muiesan, P. et al. (2015). First comparison of hypothermic oxygenated perfusion versus static cold storage of human donation after cardiac death liver transplants. Ann Surg 262, 764-771. doi:10.1097/SLA.0000000000001473

Dutkowski, P., Guarrera, J. V., de Jonge, J. et al. (2019). Evolving trends in machine perfusion for liver transplantation. Gastroenterology 156, 1542-1547. doi:10.1053/j.gastro.2018.12.037

Feng, S., Goodrich, N. P., Bragg-Gresham, J. L. et al. (2006). Characteristics associated with liver graft failure: The concept of a donor risk index. Am J Transplant 6, 783-790. doi:10.1111/j.1600-6143.2006.01242.x

Foley, D. P., Fernandez, L., Leverson, G. et al. (2011). Biliary complications after liver transplantation from donation after cardiac death donors: An analysis of risk factors and long term outcomes from a single center. Ann Surg 253, 817-825. doi:10.1097/SLA.0b013e3182104784

Grosse-Siestrup, C., Nagel, S., Unger, V. et al. (2001). The isolated perfused liver: A new model using autologous blood and porcine slaughterhouse organs. J Pharmacol Toxicol Methods 46, 163-168. doi:10.1016/S1056-8719(02)00184-3

Grosse-Siestrup, C., Fehrenberg, C., von Baeyer, H. et al. (2002a). Multiple-organ harvesting for models of isolated hemoperfused organs of slaughtered pigs. ALTEX 19, 9-13. https://www.altex.org/index.php/altex/article/view/1113/1107

Grosse-Siestrup, C., Pfeffer, J., Unger, V. et al. (2002b). Isolated hemoperfused slaughterhouse livers as a valid model to study hepatotoxicity. Toxicol Pathol 30, 749-754. doi:10.1080/01926230290166841

Guarrera, J. V., Henry, S. D., Samstein, B. et al. (2010). Hypothermic machine preservation in human liver transplantation: The first clinical series. Am J Transplant 10, 372-381. doi:10.1111/j.1600-6143.2009.02932.x

Hart, D. W., Chinkes, D. L. and Gore, D. C. (2003). Increased tissue oxygen extraction and acidosis with progressive severity of sepsis. J Surg Res 112, 49-58. doi:10.1016/S00224804(03)00141-0

Hessheimer, A. J. and Fondevila C. (2017). Liver perfusion devices: How close are we to widespread application? Curr Opin Organ Transplant 22, 105-111. doi:10.1097/MOT. 0000000000000384

Iozzo, P., Jarvisalo, M. J., Kiss, J. et al. (2007). Quantification of liver glucose metabolism by positron emission tomography: Validation study in pigs. Gastroenterology 132, 531-542. doi:10.1053/j.gastro.2006.12.040

Kim, W. R., Lake, J. R., Smith, J. M. et al. (2019). OPTN/SRTR 
2017 annual data report: Liver. Am J Transplant 19, 184-283. doi:10.1111/ajt.15276

Kramer, L., Bauer, E., Joukhadar, C. et al. (2003). Citrate pharmacokinetics and metabolism in cirrhotic and noncirrhotic critically Ill patients. Crit Care Med 31, 2450-2455. doi:10.1097/01.CCM.0000084871.76568.E6

Lonati, C., Bassani, G. A., Brambilla, D. et al. (2018). Influence of ex vivo perfusion on the biomolecular profile of rat lungs. FASEB J 32, 5532-5549. doi:10.1096/fj.201701255R

López-Navidad, A. and Caballero, F. (2003). Extended criteria for organ acceptance. Strategies for achieving organ safety and for increasing organ pool. Clin Transplant 17, 308-324. doi:10.1034/j.1399-0012.2003.00119.x

Manyalich, M., Nelson, H. and Delmonico, F. L. (2018). The need and opportunity for donation after circulatory death worldwide. Curr Opin Organ Transplant 23, 136-141. doi:10. 1097/MOT.0000000000000486

Mergental, H., Perera, M., Laing, R. W. et al. (2016). Transplantation of declined liver allografts following normothermic ex-situ evaluation. Am J Transplant 16, 3235-3245. doi:10.1111/ajt.13875

Merlen, G., Raymond, V. A., Cassim, S. et al. (2019). Oxaloacetate protects rat liver from experimental warm ischemia/reperfusion injury by improving cellular energy metabolism. Liver Transpl 25, 627-639. doi:10.1002/1t.25415

Nasralla, D., Coussios, C., Mergental, H. et al. (2018). A randomized trial of normothermic preservation in liver transplantation. Nature 557, 50-56. doi:10.1038/s41586-018-0047-9

Op den Dries, S., Karimian, N., Sutton, M. E. et al. (2013). Ex vivo normothermic machine perfusion and viability testing of discarded human donor livers. Am J Transplant 13, 13271335. doi:10.1111/ajt.12187

Quistrorff, B., Katz, N. and Witters, L. A. (1992). Hepatocyte heterogeneity in the metabolism of fatty acids: Discrepancies on zonation of acetyl-CoA carboxylase. Enzyme 46, 59-71. doi: $10.1159 / 000468778$

Ravikumar, R., Jassem, W., Mergental, H. et al. (2016). Liver transplantation after ex vivo normothermic machine preservation: A phase 1 (first-in-man) clinical trial. Am J Transplant 16, 1779-1787. doi:10.1111/ajt.13708

Roffia, V., De Palma, A., Lonati, C. et al. (2018). Proteome investigation of rat lungs subjected to ex vivo perfusion (EVLP). Molecules 23, 1-20. doi:10.3390/molecules23123061

Selten, J., Schlegel, A., de Jonge, J. et al. (2017). Hypo- and normothermic perfusion of the liver: Which way to go? Best Pract Res Clin Gastroenterol 31, 171-179. doi:10.1016/j. bpg.2017.04.001
Slimani, L., Kudomi, N., Oikonen, V. et al. (2008). Quantification of liver perfusion with [15O]H2O-PET and its relationship with glucose metabolism and substrate levels. $J$ Hepatol 48, 974-982. doi:10.1016/j.jhep.2008.01.029

Tashiro, H., Kuroda, S., Mikuriya, Y. et al. (2014). Ischemia-reperfusion injury in patients with fatty liver and the clinical impact of steatotic liver on hepatic surgery. Surg Today 44, 1611-1625. doi:10.1007/s00595-013-0736-9

Thuong, M., Ruiz, A., Evrard, P. et al. (2016). New classification of donation after circulatory death donors definitions and terminology. Transpl Int 29, 749-759. doi:10.1111/tri.12776

Verhoeven, C. J., Farid, W. R. R., de Jonge, J. et al. (2014). Biomarkers to assess graft quality during conventional and machine preservation in liver transplantation. J Hepatol 61, 672684. doi:10.1016/j.jhep.2014.04.031

Vodkin, I. and Kuo, A. (2017). Extended criteria donors in liver transplantation. Clin Liver Dis 21, 289-301. doi:10.1016/j. cld.2016.12.004

Watson, C. J. E., Kosmoliaptsis, V., Randle, L. V. et al. (2017). Normothermic perfusion in the assessment and preservation of declined livers before transplantation. Transplantation 101, 1084-1098. doi:10.1097/TP.0000000000001661

Winterdahl, M., Munk, O. L., Sørensen, M. et al. (2011). Hepatic blood perfusion measured by 3-minute dynamic 18F-FDG PET in pigs. $J$ Nucl Med 52, 1119-1124. doi:10.1038/jid.2014.371

\section{Conflict of interest}

Authors have no conflicts of interest to declare.

\section{Acknowledgements}

We proudly thank all slaughterhouse technicians and the owners of the slaughterhouse for their valuable help. They became part of our team and this paper could not have been written without their involvement. The present study was supported by Fondazione Ca' Granda Ospedale Maggiore Policlinico, Milan, with the following grants: 5x1000-2014 "Ottimizzazione della procedura di perfusione di organi isolati a scopo di trapianto mediante modulazione del liquido di perfusione - Improvement of perfusion of isolated organs before transplantation through perfusion fluid optimization", 5x1000-2014 "Il benessere animale nella ricerca preclinica: strategie di implementazione - Implementation of strategies for laboratory animal care". We also thank "Associazione Italiana Copev per la prevenzione e cura dell'epatite virale Beatrice Vitiello- ONLUS" and "MILTA". Authors would like to thank Samata Oldoni for her valuable help. 